\title{
BESS-Polar Measurements of the Cosmic-ray Proton and Helium Spectra
}

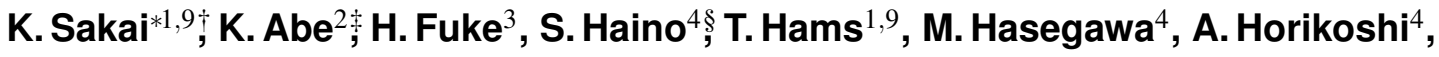 \\ A. Itazaki ${ }^{2}$, K. C. Kim ${ }^{5}$, T. Kumazawa ${ }^{4}$, A. Kusumoto ${ }^{2}$, M. H. Lee ${ }^{5}$, Y. Makida ${ }^{4}$, \\ S. Matsuda ${ }^{4}$, Y. Matsukawa ${ }^{2}$, K. Matsumoto ${ }^{4}$, J. W. Mitchell ${ }^{1}$, Z. Myers ${ }^{5}$, J. Nishimura ${ }^{6}$, \\ M. Nozaki ${ }^{4}$, R. Orito ${ }^{2 \llbracket}$ J. F. Ormes ${ }^{7}$, N. Picot-Clemente ${ }^{5}$, M. Sasaki ${ }^{1,9}$, E. S. Seo ${ }^{5}$, \\ Y.Shikaze ${ }^{2}$, R. Shinoda ${ }^{6}$, R. E. Streitmatter ${ }^{1}$, J.Suzuki ${ }^{4}$, Y. Takasugi ${ }^{2}$, K. Takeuchi ${ }^{2}$, \\ K. Tanaka ${ }^{4}$, N. Thakur ${ }^{1}$, T. Yamagami ${ }^{3}$, A. Yamamoto ${ }^{4}$, T. Yoshida ${ }^{3}$ and K. Yoshimura ${ }^{8}$ \\ ${ }^{1}$ NASA-Goddard Space Flight Center (NASA-GSFC), Greenbelt,MD 20771, USA \\ ${ }^{2}$ Kobe University, Kobe, Hyogo 657-8501, Japan \\ ${ }^{3}$ Institute of Space and Astronautical Science, Japan Aerospace Exploration Agency \\ (ISAS/JAXA), Sagamihara, Kanagawa 252-5210, Japan \\ ${ }^{4}$ High Energy Accelerator Research Organization (KEK), Tsukuba, Ibaraki 305-0801, Japan \\ 5 IPST, University of Maryland, College Park, MD 20742, USA \\ ${ }^{6}$ The University of Tokyo, Bunkyo, Tokyo 113-0033, Japan \\ ${ }^{7}$ University of Denver, Denver, CO 80208, USA \\ ${ }^{8}$ Okayama University, Okayama, Okayama 700-0082, Japan \\ ${ }^{9}$ Center for Research and Exploration in Space Science and Technology (CRESST)
}

\begin{abstract}
The energy spectra of cosmic-ray protons and helium near solar minimum were measured with BESS-Polar (Balloon-borne Experiment with a Superconducting Spectrometer-Polar) during long-duration flights over Antarctica in December 2004 and December 2007, and are discussed here. The absolute fluxes and spectral shapes of primary protons and helium probe the origin and the propagation history of cosmic rays in the Galaxy. The spectra are also essential as inputs to calculate the spectrum of cosmic-ray antiprotons, which are secondary products of cosmic-ray interactions with the interstellar gas. We report absolute spectra at the top of the atmosphere for cosmic-ray protons in the kinetic energy range $0.2-160 \mathrm{GeV}$ and helium nuclei 0.2-80 GeV/nucleon [1].
\end{abstract}

The 34th International Cosmic Ray Conference,

30 July- 6 August, 2015

The Hague, The Netherlands

\footnotetext{
* Speaker.

$\dagger$ E-mail: kenichi.sakai@nasa.gov

¥Present address: Kamioka Observatory, ICRR, The University of Tokyo, Hida, Gifu 506-1205, Japan.

${ }^{\S}$ Present address: Institute of Physics, Academia Sinica, Nankang, Taipei 11529, Taiwan.

IPresent address: Tokushima University, Tokushima, Tokushima 770-8502, Japan.

$\|_{\text {Deceased. }}$
} 


\section{Introduction}

The absolute fluxes and spectral shapes of primary cosmic rays are important for understanding the origin and the propagation history of the cosmic rays in the Galaxy. The proton and helium spectra are also essential as inputs to calculations of the spectra of cosmic-ray antiprotons and positrons, which are secondary products of cosmic-ray interactions with the interstellar gas. During propagation through the ISM, cosmic rays undergo interactions with gas atoms and loose energy, significantly modifying their spectra and composition. Then when Galactic cosmic rays enter the heliosphere, they are scattered by irregularities in the heliospheric magnetic field and undergo convection and adiabatic deceleration in the expanding solar wind. This process, modifying the energy spectra of cosmic rays, is known as "solar modulation". The abundant protons and helium nuclei are among the most important keys to understanding these processes.

\section{BESS Program}

The BESS instrument [2,3] was developed as a high-resolution magnetic-rigidity spectrometer for cosmic-ray antiparticles and precise measurements of the absolute fluxes of various cosmic-ray components. The original BESS experiment flew 8 times over Lynn Lake, Canada and once from Fort Sumner, USA during the period of 1993 through 2002 with continuous improvement in the instrument [4]. The BESS-Polar project was proposed as an advanced BESS program using long duration balloon (LDB) flights over Antarctica (around the south pole) to provide high-statistics, low-energy cosmic-ray measurements $[5,6,7]$.

The scientific flight of the BESS-Polar I instrument was launched from Williams Field, near McMurdo Station, on December 13th, 2004 (UTC). The flight duration was over 8.5 days at $37 \mathrm{~km}$ to $39.5 \mathrm{~km}$ (residual air of $4.33 \mathrm{~g} / \mathrm{cm}^{2}$ on average) and more than $9 \times 10^{8}$ cosmic-ray events were recorded. Incorporating considerable improvements in instrument and payload systems compared to BESS-Polar I, the BESS-Polar II instrument was launched on December 23, 2007, from Williams Field and circulated around the South Pole for 24.5 days of observation with the magnet energized. The float altitude was $34 \mathrm{~km}$ to $38 \mathrm{~km}$ (residual air of $5.81 \mathrm{~g} / \mathrm{cm}^{2}$ on average), and the cutoff rigidity

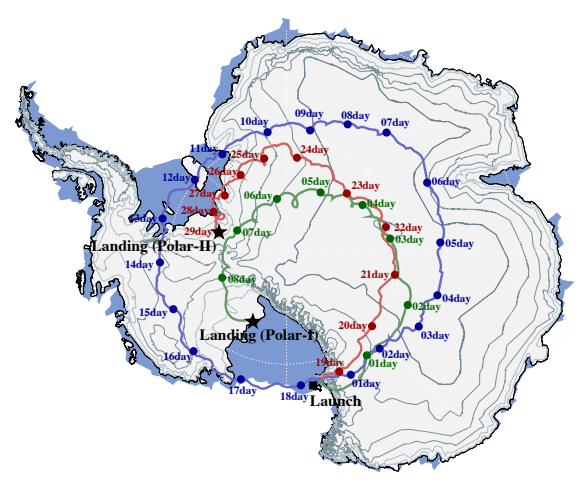

Figure 1: Flight trajectories over Antarctica of BESS-Polar I in 2004 (green) and BESS-Polar II in 2007/2008 (first orbit blue, second orbit red). 


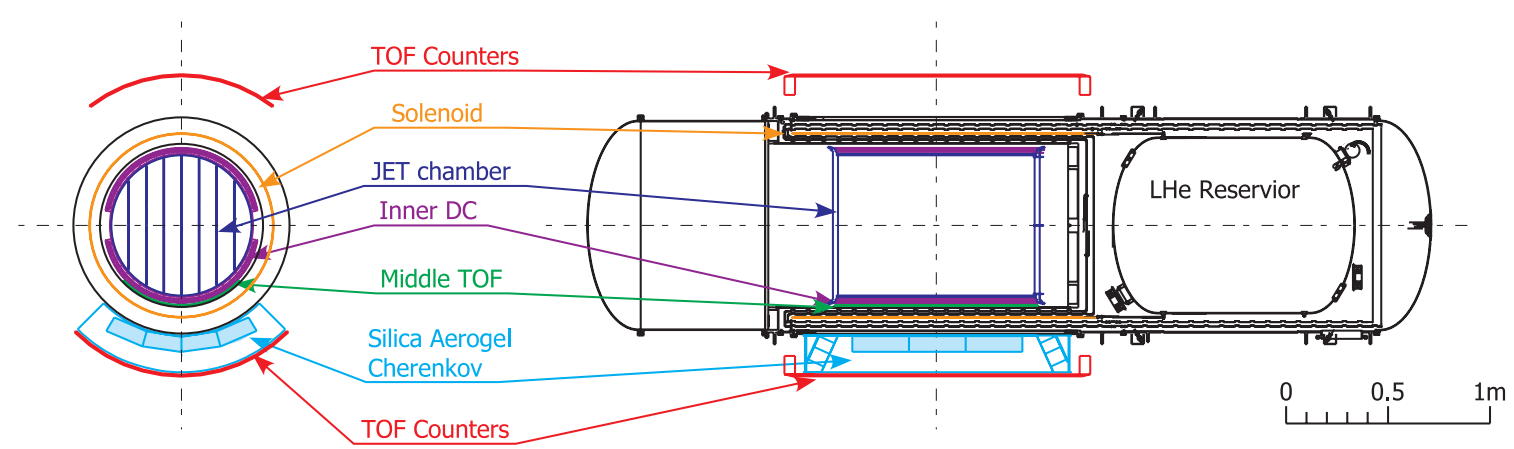

Figure 2: Cross sectional view of BESS-Polar II spectrometer

was below $0.5 \mathrm{GV}$. BESS-Polar II accumulated $4.7 \times 10^{9}$ events with no inflight event selection as 13.6 terabytes of data (Fig.1).

The BESS-Polar program has produced precise measurements of antiprotons $[8,9]$ and a sensitive antihelium search [10]. The antiproton spectrum measured by BESS-Polar II shows good consistency with secondary antiproton calculations and no evidence of primary antiprotons originating from the evaporation of primordial black holes. The antihelium work has set a new limit in the ratio of possible antihelium to measured helium of $6.9 \times 10^{-8}$ at $95 \%$ confidence, the lowest limit to date.

\section{BESS-Polar Instrument}

In the BESS-Polar instruments, a uniform field of $0.8 \mathrm{~T}$ is produced by a thin superconducting solenoid, and the field region is filled with drift-chamber tracking detectors. Tracking is performed by fitting up to 52 hit points with a characteristic resolution of $\sim 125 \mu \mathrm{m}$ in the bending plane, resulting in a magnetic-rigidity $(\equiv P c / Z e$ ) resolution of $0.4 \%$ at $1 \mathrm{GV}$ and a maximum detectable rigidity (MDR) of $240 \mathrm{GV}$. Upper and lower scintillator hodoscopes provide time-of-flight (TOF) and $d E / d x$ measurements and the event trigger with a geometric acceptance of $\sim 0.3 \mathrm{~m}^{2}$ sr. The timing resolution of the TOF system is $120 \mathrm{ps}$, giving a $\beta^{-1}$ resolution of $2.5 \%$. For antiproton measurements, the geometric acceptance of BESS-Polar is $0.23 \mathrm{~m}^{2} \mathrm{sr}$ and for proton and helium measurements the acceptance is $0.20 \mathrm{~m}^{2} \mathrm{sr}$. In BESS-Polar I, 18 of 44 PMTs in TOF had to be turned off due to excessive current and this reduced to $75 \%$ of the acceptance. 2 of 44 PMTs had HV control problems during BESS-Polar II flight; however, 100\% of the nominal acceptance was retained. The instrument also incorporates a threshold-type Cherenkov counter using a silica aerogel radiator and a thin scintillator middle-TOF. They are installed to identify antiprotons; therefore, they aren't used in this analysis for protons and helium.

\section{Solar Modulation}

The considerable variation in solar activity and details of the effects of the solar wind and its entrained magnetic fields on the incoming GCR fluxes have to be taken into account in deriving interstellar spectra. At energies above $30 \mathrm{GeV}$ for protons and $15 \mathrm{GeV}$ /nucleon for helium, solar modulation has negligible influence on the measured spectra. Figure 3 shows evidence of solar 
modulation of cosmic rays and solar activity illustrated by the changes with time of the Bartol neutron monitor counting rate (Blue points) [11] and the number of sunspots (Red points) [12] together the data of BESS flights. The solar cycle has an approximately 11-year period. In addition, the sun has a 22 year magnetic cycle with recurrent positive $(A>0)$ and negative $(A<0)$ phases, where $A<0$ polarity cycles are defined as the periods when the heliospheric magnetic field (HMF) is directed towards the Sun in the northern hemisphere. Since the low-energy region of the cosmic radiation is most intensively affected by solar modulation, the absolute fluxes and spectral shapes of primary protons and helium obtained with BESS are essential probes. These are combined with the simultaneous BESS antiproton measurements to probe the effect of charge-sign dependent drift on the entering cosmic rays.

\section{Data analysis}

In the first stage of data analysis, we selected events with a single track fully contained inside the fiducial volume defined by the central four columns out of eight columns in the JET chamber. To ensure the best tracking, an effective IDC fiducial region of about $\sim 85 \%$ of the active area was defined by cuts on the drift time to exclude regions very near the sense wires and far from the wires. These definitions of the fiducial volume reduced the effective geometrical acceptance, but ensured the longest track fits and thus the highest resolution in the rigidity measurement. The effective acceptances at $10 \mathrm{GeV} /$ nucleon in BESS-Polar I are $0.042 \mathrm{~m}^{2} \mathrm{sr}$ for protons and $0.038 \mathrm{~m}^{2} \mathrm{sr}$ for helium. In BESS-Polar II they are $0.056 \mathrm{~m}^{2} \mathrm{sr}$ for protons and $0.051 \mathrm{~m}^{2} \mathrm{sr}$ for helium. A singletrack event was defined as an event which has only one isolated track and one or two hit counters in each layer of the TOF hodoscopes. The single-track selection eliminated rare interacting events.

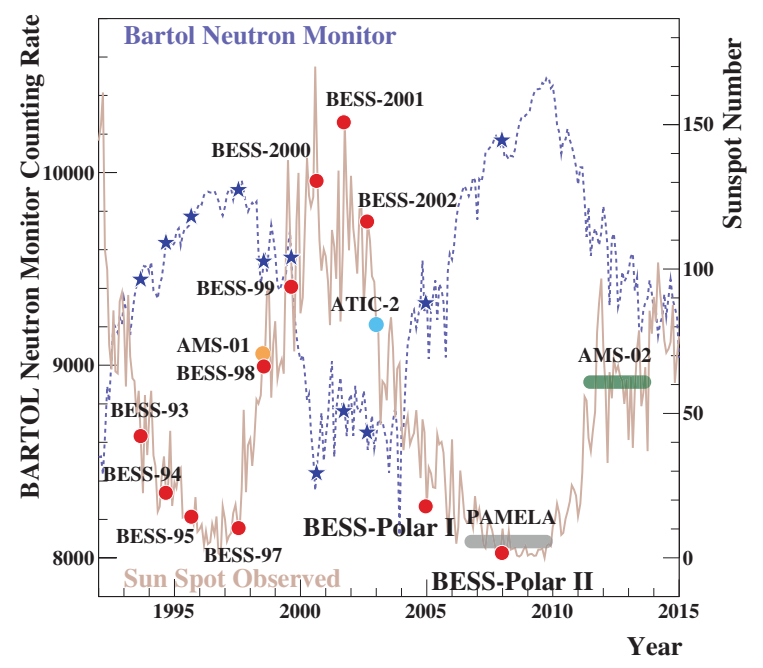

Figure 3: Variation of neutron monitor and sunspot number together the data of the BESS, AMS-01, ATIC2, PAMELA, AMS-02 and BESS-Polar flights. The BESS-Polar II flight was carried out near the absolute solar minimum. 

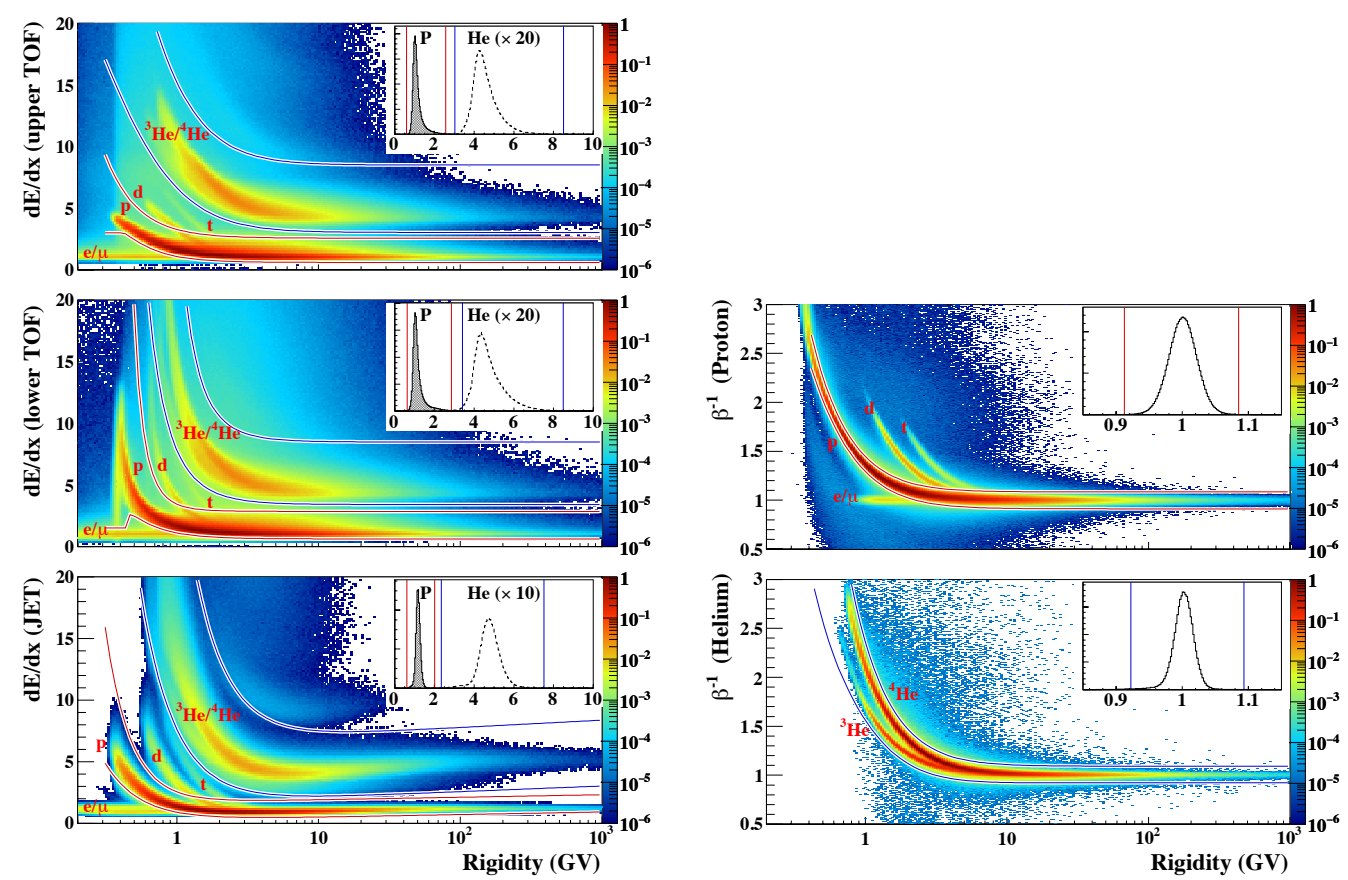

Figure 4: Proton and helium bands in $\mathrm{dE} / \mathrm{dx}$ Figure 5: Scatter plot of $\beta^{-1}$ vs. rigidity (top: upper TOF, middle: lower TOF, bottom: obtained from the balloon observation after JET) vs. rigidity obtained from the balloon proton (top) and helium (bottom) dE/dx observation. The superimposed graph shows the selection. The superimposed graph shows the selection criteria for protons and helium nuclei selection criteria for protons and helium nuclei above $30 \mathrm{GV}$. above $30 \mathrm{GV}$.

To estimate the efficiency of the single-track selection, Monte Carlo simulations with GEANT4 (Version 10.00.p01) were performed.

\subsection{Event selection}

To assure the accuracy of the rigidity measurement, the following track quality cuts were applied: (1) reduced $\chi^{2}$ per degree of freedom $<5$ for track fits in both $r \phi$ and $y z$ planes (where $y$ is the vertical direction), (2) track fitting pathlength $\geq 500 \mathrm{~mm}$, (3) ratio of fitted hits in the JET to expected hits $>0.6$, (4) ratio of dropped hits in the JET and IDCs to expected hits $<0.25$, (5) 2 hits in each of the 2 IDCs all giving both $x$ and $z$ positions, and (6) consistency between independent $z$ positions measured by the tracking system and the TOF (determined using the time difference between each end of the hit paddle). Above $\sim 2 \mathrm{GV}$ the efficiencies for both protons and helium are essentially constant. Particle identification was performed by requiring proper $\mathrm{dE} / \mathrm{dx}$ measurements, in both upper and lower layers of the TOF hodoscopes, and $\beta^{-1}$ as functions of rigidity. Figs. 4 and 5 show the selection criteria for protons and helium. The efficiencies of $\mathrm{dE} / \mathrm{dx}$ selection were estimated with another sample selected by independent measurement of energy loss inside the JET. Since the $\beta^{-1}$ distribution is well described by Gaussian and a halfwidth of the $\beta^{-1}$ selection band was set at $4 \sigma$ for protons and $6 \sigma$ for helium, the efficiency is very close to unity. 


\subsection{Normalization and corrections}

$$
\begin{array}{r}
\Phi_{\mathrm{TOA}}\left(E_{\mathrm{TOA}}\right)=\frac{\Phi_{\mathrm{TOI}}\left(E_{\mathrm{TOI}}\right)}{\eta\left(E_{\mathrm{TOI} \rightarrow \mathrm{TOA}}\right)+R_{\mathrm{air}}\left(E_{\mathrm{TOI} \rightarrow \mathrm{TOA}}\right)} \\
\Phi_{\mathrm{TOI}}\left(E_{\mathrm{TOI}}\right)=N_{\mathrm{p}} /\left(\varepsilon_{\mathrm{det}} \cdot \varepsilon_{\mathrm{MC}}\right) /\left(S \Omega \cdot T_{\text {live }}\right)
\end{array}
$$

where $N_{\mathrm{p}}$ is number of observed particles of that species, $\varepsilon_{\text {det }}$ is detection efficiency that is related to the event selection, $\varepsilon_{\mathrm{MC}}$ is noninteracted single track efficiency estimated by using MC, $S \Omega$ is geometrical acceptance and $T_{\text {live }}$ is the live time period. $(1-\eta)$ quantifies losses in the atmosphere and $R_{\text {air }}$ is atmospheric secondary production.

In order to determine the primary cosmic-ray proton and helium spectra at the top of the atmosphere, the following normalization and corrections are required : (1) exposure factor, (2) ionization energy loss, (3) interaction loss, and (4) atmospheric secondary particle contribution.

The exposure factor is a product of geometrical acceptance and live time. The geometrical acceptance defined for this analysis was calculated by simulation technique described in Section 5. The simple cylindrical shape and the uniform magnetic field make it simple and reliable to determine the geometrical acceptance precisely. The live data-taking time was measured to be $5 \times 10^{5} \mathrm{~s}$ in BESS-Polar I and $1.3 \times 10^{6} \mathrm{~s}$ in BESS-Polar II. The energy of each incoming particle was calculated by integrating the energy losses inside the detector tracing back along the particle trajectory. In order to obtain the absolute flux of primary protons and helium nuclei at the top of the atmosphere, interaction loss and secondary particle production in the residual atmosphere were estimated. Both for air correction can be estimated by solving simultaneous transport equations following Papini et al [13]. The primary spectrum at TOA $\left(\Phi_{\mathrm{TOA}}\right)$ is determined in an iterative procedure so that the estimated spectrum at TOI $\left(\Phi_{\mathrm{TOI}}\right)$ agrees with the observed one.

\section{Measured Spectra}

In the present work, we have obtained the absolute fluxes of primary protons in the range 0.2 $160 \mathrm{GeV}$ and helium nuclei in the range $0.2-80 \mathrm{GeV} /$ nucleon at the top of the atmosphere from BESS-Polar I and BESS-Polar II balloon-flight data, respectively. The corresponding ranges are 0.6-160 GV for protons and 1.3-160 GV for helium. The overall uncertainties, including both statistical and systematic errors, are less than $\pm 10 \%$ for BESS-Polar I protons, $\pm 6 \%$ for BESSPolar II protons, $\pm 7 \%$ for BESS-Polar I helium, and $\pm 5 \%$ for BESS-Polar II helium. The results of primary proton and helium spectra are shown in Fig. 6 in comparison with BESS98 [14], AMS01 [15, 16], BESS-TeV [17], ATIC-2 [18], PAMELA [19], and AMS-02 [20, 21].

Differences in the spectra measured by BESS-Polar I and BESS-Polar II resulting from solar activity (discussed in Section 4) below $\sim 10 \mathrm{GeV}$ for protons and below $\sim 5 \mathrm{GeV}$ /nucleon for helium are highlighted. This variation of measured spectra is consistent with the effect expected from charge-sign dependent drift [22, 23]. At energies above $30 \mathrm{GeV}$ for protons and $15 \mathrm{GeV} /$ nucleon for helium, the effects of solar modulation are effectively negligible and the proton spectra measured BESS-Polar I and BESS-Polar II are essentially identical, differing by less than $1 \%$ at $160 \mathrm{GeV}$ despite having been obtained during quite different periods of solar activity (see further discussion 


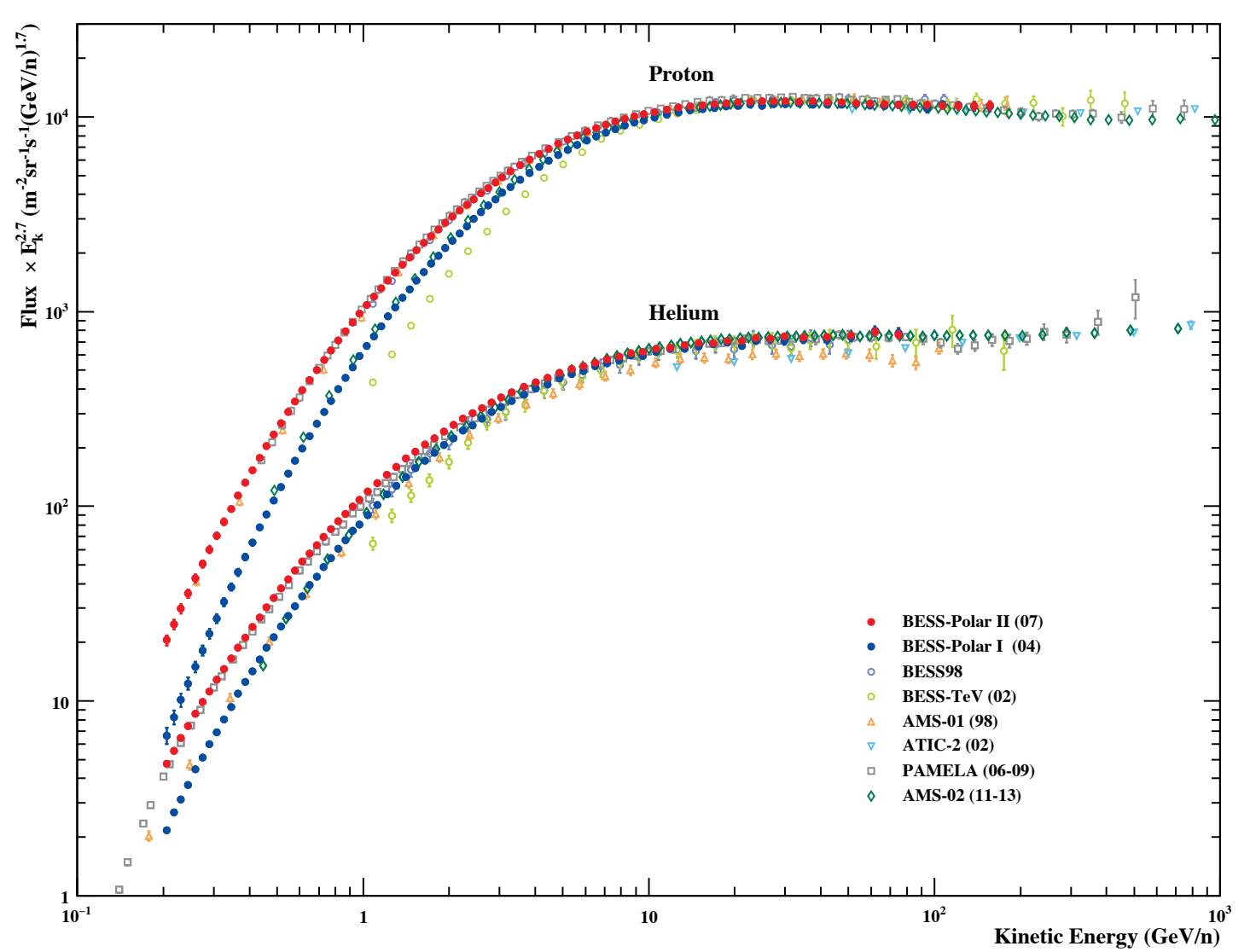

Figure 6: Absolute differential energy spectrum of primary protons and helium multiplied by $E_{K}^{2.7}$ measured by BESS-Polar I and BESS-Polar II. The spectra obtained by other experiments [14, 15, 16, 17, 18, 19, 20, 21] are also shown.

below). The same is true of the helium spectra above $\sim 15 \mathrm{GeV} /$ nucleon where the BESS-Polar I and BESS-Polar II results differ by less than $1 \%$ at $80 \mathrm{GeV} /$ nucleon.

\section{Conclusion}

We have measured energy spectra of primary protons in the range $0.2-160 \mathrm{GeV}$ and helium nuclei in the range 0.2-80 GeV/nucleon at the top of the atmosphere from BESS-Polar I and BESSPolar II. Full details of the analysis are described in a paper submitted to arXiv[1].

\section{Acknowledgements}

The BESS-Polar program is a Japan-United States collaboration, supported in Japan by the Grant-in-Aid 'KAKENHI' for Specially Promoted and Basic Researches, MEXT-JSPS, and in the U.S. by NASA. Balloon flight operations were carried out by the NASA Columbia Scientific Balloon Facility and the National Science Foundation United States Antarctic Program. We would like to express our sincere thanks for their continuous professional support. 


\section{References}

[1] K. Abe et al., Measurements of cosmic-ray proton and helium spectra from the BESS-Polar long-duration balloon flights over Antarctica, arXiv:1506.0126.

[2] BESS Collaboration, Y. Ajima et al., A superconducting solenoidal spectrometer for a balloon-borne experiment, Nucl. Instrum. Meth. A443 (2000) 71.

[3] S. Haino et al., Progress of the BESS superconducting spectrometer, Nucl. Instrum. Meth. A518 (2004) 167.

[4] J. Mitchell et al. the 33rd International Cosmic Ray Conference (Rio de Janeiro, Brazil, 2013), ID.1087 (2013).

[5] A. Yamamoto et al., BESS and its future prospect for polar long duration flights, Adv. Space Res. 30 (2002) 1253.

[6] J. W. Mitchell et al., The BESS program, Nucl. Phys. (Proc. Suppl.) 134 (2004) 31.

[7] T. Yoshida et al., BESS-polar experiment, Adv. Space Res. 33 (2004) 1755.

[8] K. Abe et al., Measurement of cosmic-ray low-energy antiproton spectrum with the first BESS-Polar Antarctic flight, Phys. Lett. B670 (2008) 103, [arXiv: 0805.1754$].$

[9] K. Abe et al., Measurement of the cosmic-ray antiproton spectrum at solar minimum with a long-duration balloon flight over antarctica, Phys. Rev. Lett. 108 (Jan, 2012) 051102.

[10] K. Abe et al., Search for antihelium with the bess-polar spectrometer, Phys. Rev. Lett. 108 (Mar, 2012) 131301.

[11] ftp://ftp.bartol.udel.edu/pyle/BRIData/.

[12] http://solarscience.msfc.nasa.gov/greenwch/.

[13] P. Papini, C. Grimani, and S. A. Stephens, An estimate of the secondary-proton spectrum at small atmospheric depths, Nuovo Cim. C19 (1996) 367.

[14] T. Sanuki et al., Precise measurement of cosmic-ray proton and helium spectra with the bess spectrometer, The Astrophysical Journal 545 (2000), no. 21135.

[15] J. Alcaraz et al., Cosmic protons, Phys. Lett. B 490 (2000), no. 1-2 27 - 35.

[16] J. Alcaraz et al., Helium in near earth orbit, Phys. Lett. B 494 (2000), no. 3-4 193 - 202.

[17] S. Haino et al., Measurements of primary and atmospheric cosmic-ray spectra with the BESS-TeV spectrometer, Phys. Lett. B594 (2004) 35, [a stro-ph/ 0403704 ].

[18] A. Panov et al., Elemental energy spectra of cosmic rays from the data of the atic-2 experiment, Bull. Russ. Acad. Sci. Phys. 71 (2007), no. 4 494-497.

[19] O. Adriani et al., Pamela measurements of cosmic-ray proton and helium spectra, Science 332 (2011), no. 6025 69-72, [http: / /www.sciencemag.org/content/332/6025/69.full.pdf].

[20] AMS Collaboration Collaboration, M. Aguilar et al., Precision measurement of the proton flux in primary cosmic rays from rigidity $1 \mathrm{gv}$ to $1.8 \mathrm{tv}$ with the alpha magnetic spectrometer on the international space station, Phys. Rev. Lett. 114 (Apr, 2015) 171103.

[21] S. Haino AMS Days at CERN (Geneva, Switzerland, 2015) (2015).

[22] J. W. Bieber et al., Antiprotons at solar maximum, Phys. Rev. Lett. 83 (Jul, 1999) 674-677.

[23] Y. Asaoka et al., Measurements of cosmic-ray low-energy antiproton and proton spectra in a transient period of solar field reversal, Phys. Rev. Lett. 88 (Jan, 2002) 051101. 